\title{
Preparation and the Microwave and Infrared Spectra of Vinyl Ketene
}

\author{
Erik Bjarnov* \\ Physikalisch-Chemisches Institut, Justus-Liebig-Universität Gießen, Gießen
}

Z. Naturforsch. 34a, 1269-1274 (1979); received September 27, 1979

\begin{abstract}
Vinyl ketene (1,3-butadiene-1-one) has been synthesized by vacuum pyrolysis of 3-butenoic 2-butenoic anhydride. The microwave and infrared spectra of vinyl ketene in the gas phase at room temperature have been studied. The trans-rotamer has been identified, and the spectroscopic constants were found to be $\tilde{A}=39571(48) \mathrm{MHz}, \tilde{B}=2392.9252(28) \mathrm{MHz}, \tilde{C}=$ $2256.0089(28) \mathrm{MHz}, \Delta_{J}=0.414(31) \mathrm{kHz}$, and $\Delta_{J K}=-34.694(92) \mathrm{kHz}$. The electrical dipole moment was found to be $0.987(23) D$ with $\mu_{a}=0.865(14) D$ and $\mu_{b}=0.475(41) D$. A tentative assignment has been made for 17 of the 21 normal modes of vibration.
\end{abstract}

\section{Introduction}

Vinyl ketene (1,3-butadiene-1-one) has been proposed as an intermediate from the dehydrochlorination of trans-2-butenoyl chloride [1]. Rousseau et al. have proposed a red compound, stable up to $-160^{\circ} \mathrm{C}$, from the flash thermolysis of spiro[2,3]hexan-4-ones to be the free vinyl ketene, since the reaction with methanol gave methyl vinylacetate [2]. Very recently Terlouw et al. have reported that the major product from the gas phase thermal decomposition of ethyl but-3-ene1 -yne ether is vinyl ketene identified from its mass spectrum and photoelectron spectrum [3]. However, the present study appears to be the first where vinyl ketene has been isolated and unambiguously identified. The microwave and infrared spectra of vinyl ketene will be presented and a tentative vibrational assignment will be proposed.

Vinyl ketene can have two planar rotamers of which the trans configuration shown in Fig. 1 is expected to be the more abundant one at room temperature. At present only the trans-rotamer has been observed in the microwave spectrum and the major features in the infrared spectrum can be explained on the basis of just one rotamer. In the structurally related molecule vinyl allene $(1,2,4$ pentatriene) [4] only the trans-rotamer has been observed so far, while for vinyl isocyanate [5] and vinyl azide (6] both the trans and cis configurations have been reported.

\footnotetext{
* Present address and reprint requests to Dr. E. Bjarnov, Department of Chemistry, Michigan State University, East Lansing, MI 48824, USA.
}

\section{Experimental Procedures}

Vinyl ketene was first found in this laboratory in the products from the vacuum pyrolysis of trans2-butenoic anhydride (crotonic anhydride from E. Merck, Darmstadt), but the use of the mixed 3 -butenoic 2-butenoic anhydride as a precursor gave better yields. The mixed anhydride was prepared from 3-butenoic acid and trans-2-butenoyl chloride (both from E. Merck, Darmstadt) using standard methods. The pyrolysis proceeded as follows: after evacuation of the system the precursor was left at room temperature and its vapors passed

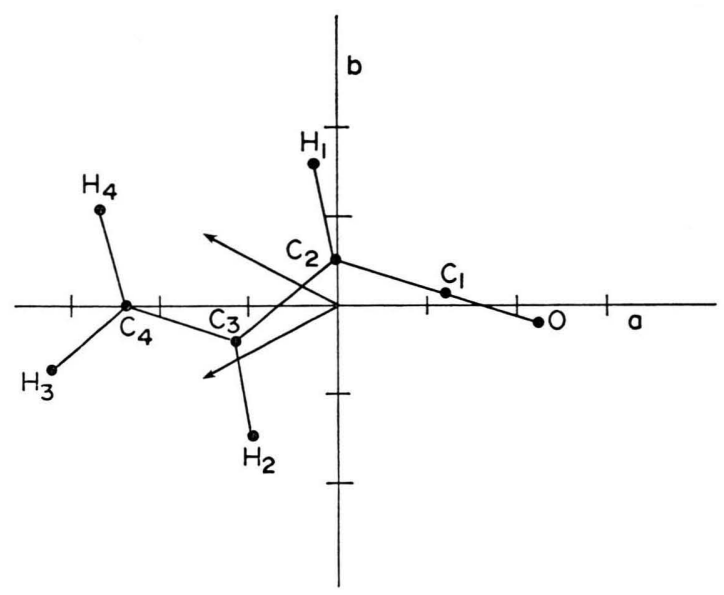

Fig. 1. Assumed structure of trans-vinyl ketene in the principal axis system. Distance are: $\mathrm{C}_{1}=\mathrm{O} \quad 1.171 \AA$, $\mathrm{C}_{2}=\mathrm{C}_{1} 1.306 \AA, \mathrm{C}_{3}-\mathrm{C}_{2} 1.43 \AA, \mathrm{C}_{4}=\mathrm{C}_{3} 1.335 \AA$, and all $\mathrm{C}-\mathrm{H} 1.088 \AA$. Angles are $\mathrm{C}_{1} \mathrm{C}_{2} \mathrm{H}_{1}=117^{\circ}, \mathrm{C}_{1} \mathrm{C}_{2} \mathrm{C}_{3}=$ $\mathrm{C}_{2} \mathrm{C}_{3} \mathrm{C}_{4}=122.5^{\circ}, \quad \mathrm{C}_{1} \mathrm{C}_{3} \mathrm{H}_{2}=\mathrm{C}_{3} \mathrm{C}_{4} \mathrm{H}_{3}=\mathrm{H}_{3} \mathrm{C}_{4} \mathrm{H}_{4}=120^{\circ}$. The arrows indicate the positive ends of the two possible directions of the electric dipole moment. 
through a quartz tube of $12 \mathrm{~mm}$ inner diameter, heated to $520^{\circ} \mathrm{C}$ over $20 \mathrm{~cm}$. Following the quartz tube was a cold trap at $-65^{\circ} \mathrm{C}$ and one at $-196^{\circ} \mathrm{C}$. The product in the $-196^{\circ} \mathrm{C}$ trap was purified by trap-to-trap distillation at $-85^{\circ} \mathrm{C}$ until the infrared spectrum showed no further changes. The first fractions mainly consisted of carbon dioxide, propyne, and some propene.

Vinyl ketene has a greenish yellow color in the solid and the liquid phases. It melts at $-85 \pm 10^{\circ} \mathrm{C}$. It polymerizes slowly in the cold melt and rapidly in the gas phase at room temperature at pressures above ca. 5 Torr. There are some indications that the gas phase polymerization is autocatalytic.

The microwave spectra in the region 8000 to $30,000 \mathrm{MHz}$ were recorded at room temperature with a Hewlett Packard 8460 A MRR spectrometer. The electric field strength in the Stark cell was calibrated using the dipole moment of OCS [7], $\mu=0.71521 D$. Vinyl ketene decomposed slowly in the Stark cell.

Infrared spectra in the region $4000-450 \mathrm{~cm}^{-1}$ were recorded on a Digilab FTS 20 Fourier spectrometer using $10 \mathrm{~cm}$ and $3 \mathrm{~m}$ gas cells. Since vinyl ketene polymerizes at low (5 Torr) pressures, only relatively weak spectra have been obtained.

\section{Microwave Spectrum}

The microwave spectrum of vinyl ketene shows patterns typical for a slightly asymmetric prolate top rotor $(x=-0.99)$ with low-lying vibrational states. Figure 2 shows the $J=5 \leftarrow 4$ a-type $\mathrm{R}$-branch transitions. The observed frequencies for the transitions of the ground vibrational state are given in Table 1. Table 2 gives the spectroscopic
Table 1. Observed and calculated frequencies in $\mathrm{MHz}$ of trans-Vinylketene in the vibrational ground state.

\begin{tabular}{lll}
\hline Transition & $\begin{array}{l}\text { Observed } \\
\text { frequency }\end{array}$ & $\begin{array}{l}\text { Calculated } \\
\text { frequency }\end{array}$ \\
\hline & 9297.474 & $9297.478(6)^{\mathrm{a}}$ \\
$2_{0,2}-1_{0,1}$ & 9161.069 & $9161.078(7)$ \\
$2_{1,2}-1_{1,1}$ & 9434.917 & $9434.910(7)$ \\
$2_{1,1}-1_{1,0}$ & 13945.250 & $13945.248(7)$ \\
$3_{0,3}-2_{0,2}$ & 13741.350 & $13741.356(10)$ \\
$3_{1,3}-2_{1,2}$ & 14152.105 & $14152.104(10)$ \\
$3_{1,2}-2_{1,1}$ & 13947.695 & $13947.590(7)$ \\
$3_{2,2}-2_{2,1}$ & 13949.040 & $13949.100(7)$ \\
$3_{2,1}-2_{2,0}$ & & \\
$4_{0,4}-3_{0,3}$ & 18591.850 & $18591.856(8)$ \\
$4_{1,4}-3_{1,3}$ & 18321.315 & $18321.323(11)$ \\
$4_{1,3}-3_{1,2}$ & 18868.985 & $18868.984(11)$ \\
$4_{2,3}-3_{2,2}$ & 18596.455 & $18596.447(7)$ \\
$4_{2,2}-3_{2,1}$ & 18600.210 & $18600.222(8)$ \\
$4_{3,2}-3_{3,1}$ & 18598.880 & $18598.885(8)$ \\
$4_{3,1}-3_{3,0}$ & 18598.880 & $18598.881(8)$ \\
$5_{0,5}-4_{0,4}$ & 23236.910 & $23236.915(11)$ \\
$5_{1,5}-4_{1,4}$ & 22900.870 & $22900.875(12)$ \\
$5_{1,4}-4_{1,3}$ & 23585.440 & $23585.443(14)$ \\
$5_{2,4}-4_{2,3}$ & 23245.020 & $23245.013(6)$ \\
$5_{2,3}-4_{2,2}$ & 23252.555 & $23252.561(11)$ \\
$5_{3,3}-4_{3,2}$ & 23248.880 & \\
$5_{3,2}-4_{3,1}$ & 23248.880 & $23248.891(6)$ \\
$5_{4}-4_{4,1}$ & 23250.850 & \\
$5_{4,1}-4_{4,0}$ & 23250.850 & $23250.854(11)$ \\
$6_{0,6}-5_{0,5}$ & 27880.030 & $27880.039(19)$ \\
$6_{1,6}-5_{1,5}$ & 27479.930 & $27479.913(16)$ \\
$6_{1,5}-5_{1,4}$ & & $28301.374(20)$ \\
$6_{2,5}-5_{2,4}$ & 27893.225 & $27893.214(10)$ \\
$6_{2,4}-5_{2,3}$ & 27906.430 & $27906.411(19)$ \\
$6_{3,4}-5_{3,3}$ & 27899.065 & $27899.052(9)$ \\
$6_{3,3}-5_{3,2}$ & 27899.065 & $27899.088(9)$ \\
$6_{4,3}-5_{4,2}$ & 27901.180 & $27901.192(12)$ \\
$6_{4,2}-5_{4,1}$ & 27901.180 & $27901.192(12)$ \\
$6_{5,2}-5_{5,1}$ & 27904.610 & $27904.599(20)$ \\
$6_{5,1}-5_{5,0}$ & 27904.610 & $27904.599(20)$ \\
\hline & & \\
\hline
\end{tabular}

a Numbers in parentheses are one standard error in units of the last digit.

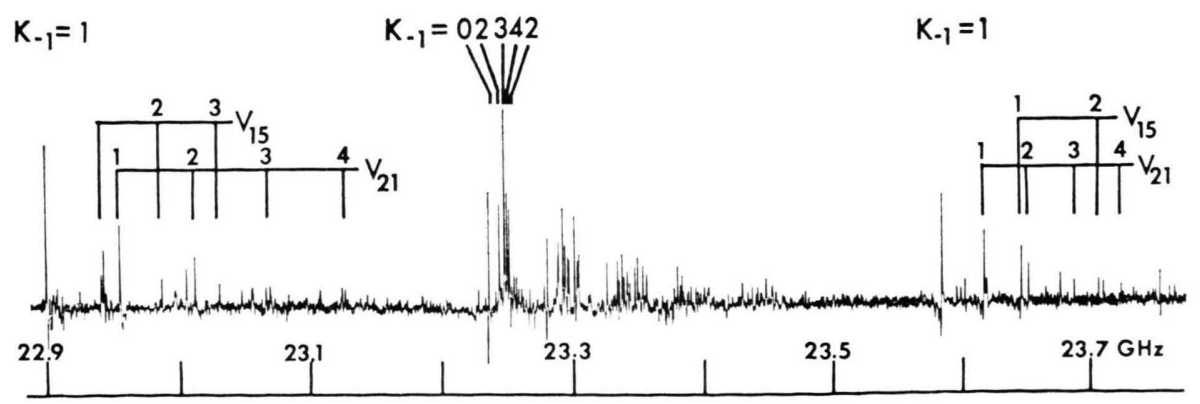

Fig. 2. The microwave spectrum of the $J=5 \leftarrow-4$ transitions of trans-vinyl ketene at room temperature. The intensity difference between the two ground state $K_{-1}=1$ lines illustrates the decay of vinyl ketene in the microwave cell in ca. 25 minutes. 
Table 2. Spectroscopic constants and moments of inertia of trans-Vinylketene.

\begin{tabular}{lccl}
\hline & Experimental & Predicted $^{\mathrm{a}}$ & \\
\hline$\tilde{A}$ & $39571(48)^{\mathrm{b}}$ & 40181 & $\mathrm{MHz}$ \\
$\tilde{B}$ & $2392.9252(28)$ & 2439 & $\mathrm{MHz}$ \\
$\tilde{C}$ & $2256.0089(28)$ & 2300 & $\mathrm{MHz}$ \\
$\Delta_{J}$ & $0.414(31)$ & & $\mathrm{kHz}$ \\
$\Delta_{J K}$ & $-34.694(92)$ & & $\mathrm{kHz}$ \\
$\sigma^{\mathrm{c}}$ & 26.5 & & $\mathrm{kHz}$ \\
$I_{a} \mathrm{~d}$ & $12.771(16)$ & 12.58 & $\mathrm{u} \AA^{2}$ \\
$I_{b}$ & $211.197(16)$ & 207.17 & $\mathrm{u} \AA^{2}$ \\
$I_{c}$ & $224.015(16)$ & 219.75 & $\mathrm{u} \AA^{2}$ \\
$I_{c}-I_{a}-I_{b}$ & $0.047(23)$ & 0.0 & $\mathrm{u} \AA^{2}$ \\
\hline
\end{tabular}

a Calculated from the assumed structure, Figure 1. The expected rotational constants for the corresponding cis-rotamer are $A=13905 \mathrm{MHz}, B=3582 \mathrm{MHz}$ and $C=2848 \mathrm{MHz}$.

b Numbers in parentheses represent one standard error in units of the last digit.

c Standard deviation of the fit.

d Calculated using the conversion factor $B^{*} I_{b}=505379.0(38) \mathrm{MHz} \cdot \mathrm{u} \AA^{2}$.

constants obtained by fitting these transitions to Watson's reduced Hamiltonian [8]:

$$
\begin{aligned}
\mathscr{H}= & \frac{1}{2}(\tilde{B}+\tilde{C}) P^{2}+\left[\tilde{A}-\frac{1}{2}(\tilde{B}+\tilde{C})\right] P_{a}^{2} \\
& +\frac{1}{2}(\tilde{B}-\tilde{C})\left(P_{b}^{2}-P_{c}^{2}\right) \\
& -\Delta_{J} P^{4}-\Delta_{J K} P^{2} P_{a}^{2}-\Delta_{K} P_{a}^{4} \\
& -2 \delta_{J} P^{2}\left(P_{b}^{2}-P_{c}^{2}\right) \\
& -\delta_{K}\left[P_{a}^{2}\left(P_{b}^{2}-P_{c}^{2}\right)+\left(P_{b}^{2}-P_{c}^{2}\right) P_{a}^{2}\right]
\end{aligned}
$$

where $P, P_{a}, P_{b}$ and $P_{c}$ are the operators for the total angular momentum and its components along the principal inertial axes. $\tilde{A}, \widetilde{B}$ and $\widetilde{C}$ are Watson's reduced rotational constants and the deltas are quartic centrifugal distortion constants. Due to the limited data $\Delta_{K}, \delta_{J}$ and $\delta_{K}$ were fixed at zero. The small, positive inertial defect $I_{c}-I_{a}-I_{b}$ confirms that the molecule is planar. An approximate structure of trans-vinyl ketene is shown in Figure 1. The rotational constants calculated from this structure are also given in Table 2 . The good agreement with the experimental rotational constants confirm that it is trans-vinyl ketene.

The observed and calculated Stark coefficients and the electric dipole moment of trans-vinyl ketene are shown in Table 3 . The two possible orientations of the dipole moment are shown in Figure 1. It may be expected that the dipole moment is directed along the ketene group just as it is in methyl ketene [9].
Table 3. Stark coefficients and dipole moment of transvinyl ketene.

\begin{tabular}{llcc}
\hline Transition & $|M|$ & $\Delta v / E^{2}$ (obs.) ${ }^{\mathrm{a}}$ & $\Delta v / E^{2}$ (calc.) \\
\hline $4_{0,4}-3_{0,3}$ & 1 & -0.280 & -0.283 \\
& 2 & 0.205 & 0.207 \\
$4_{1,4}-3_{1,3}$ & 0 & -0.114 & -0.121 \\
& 1 & 1.264 & 1.274 \\
$5_{1,5}-4_{1,4}$ & 2 & 5.461 & 5.460 \\
& 0 & 0.141 & 0.138 \\
& 1 & 0.427 & 0.425 \\
& 2 & 1.280 & 1.286 \\
$\mu_{a}{ }^{2}=0.749(24)$ & $D^{2} \mathrm{~b}$ & $\mu_{a}=0.865(14) D$ \\
$\mu_{b}{ }^{2}=0.226(39)$ & $D^{2}$ & $\mu_{b}=0.475(41)$ & $D$ \\
$\mu_{\text {total }}=0.987(23)$ & $D$ & & \\
\hline
\end{tabular}

a The units are $\mathrm{MHz} \cdot \mathrm{V}^{-2} \cdot \mathrm{cm}^{2}$.

b Numbers in parentheses represent the estimated error in units of the last digit.

A few transitions of vinyl ketene in the assumed $v_{21}=1$ and $v_{15}=1$ vibrational states were measured in order to confirm the vibrational assignment. The transitions were chosen to get the maximum A-dependence and a minimum centrifugal distortion contribution. These transitions were used to calculate the rigid rotor rotational constants. For comparison the corresponding ground state transitions were treated in the same way. Table 4 lists for the three states the transitions used, the rigid rotor rotational constants, the moments of inertia and the inertial defects. Since $v_{21}$, the vinyl torsional vibration, is an out-of-plane vibration it is expected that the inertial defect for the $v_{21}=1$ state is

Table 4. Selected transitions of trans-vinyl ketene in the ground, the $v_{21}=1$ and the $v_{15}=1$ vibrational states. Rigid rotor rotational constants ${ }^{\mathrm{a}}$, moments of inertia and inertial defects for these states.

\begin{tabular}{lcccl}
\hline & $\begin{array}{l}\text { Ground } \\
\text { state }\end{array}$ & $v_{21}=1$ & $v_{15}=1$ & \\
\hline $5_{1,4}-4_{1,3}$ & 23585.440 & 23618.330 & 23647.255 & $\mathrm{MHz}$ \\
$5_{1,5}-4_{1,4}$ & 22900.870 & 22957.280 & 22944.240 & $\mathrm{MHz}$ \\
$5_{2,3}-4_{2,2}$ & 23252.555 & 23297.250 & 23305.570 & $\mathrm{MHz}$ \\
$5_{2,4}-4_{2,3}$ & 23245.020 & 23289.595 & 23298.220 & $\mathrm{MHz}$ \\
$A$ & 39641 & 36580 & 42686 & $\mathrm{MHz}$ \\
$B$ & 2392.9 & 2395.1 & 2400.0 & $\mathrm{MHz}$ \\
$C$ & 2256.0 & 2262.8 & 2259.4 & $\mathrm{MHz}$ \\
$I_{a}$ & 12.75 & 13.82 & 11.84 & $\mathrm{u} \AA^{2}$ \\
$I_{b}$ & 211.20 & 211.01 & 210.57 & $\mathrm{u} \AA^{2}$ \\
$I_{c}$ & 224.02 & 223.34 & 223.68 & $\mathrm{u} \AA^{2}$ \\
$I_{c}-I_{a}-I_{b}$ & 0.07 & -1.49 & 1.27 & $\mathrm{u} \AA^{2}$ \\
\hline
\end{tabular}

a Calculated from the transitions listed using the rigid rotor tables in C. H. Townes and A. L. Schawlow, Microwave Spectroscopy, McGraw-Hill, New York 1955. 
negative and the inertial defect for the $v_{21}=1$ state is actually found to be $-1.5 \mathrm{u} \AA^{2}$. Thus the inertial defects confirm the vibrational assignment that $v_{21}$ is the lowest vibration of vinyl ketene with an in-plane bending mode, $v_{15}$, nearby.

There are still many unassigned lines in the microwave spectra. Some of these stem undoubtedly from decomposition products and many arise from molecules in excited vibrational states, but none of the lines could be confidently assigned either to $b$-type transitions of the trans-rotamer or to transitions of the cis-rotamer.

\section{Infrared Spectra and Assignment}

The gas phase infrared spectrum of vinyl ketene in the range $3600-400 \mathrm{~cm}^{-1}$ is shown in Figure 3 . A proof of the identity of vinyl ketene is given by the intensity and wavenumber of a very strong band at $2137 \mathrm{~cm}^{-1}$, just $14 \mathrm{~cm}^{-1}$ below that of ketene itself [10], strongly indicating the presence of a ketene group. The vinyl group is indicated by a strong band at $1626 \mathrm{~cm}^{-1}$ and two out of plane vibrations at 976 and $875 \mathrm{~cm}^{-1}$ [11]. Since vinyl ketene has only $C_{\mathrm{s}}$ symmetry the band contours of in-plane vibrations will be $\mathrm{A} / \mathrm{B}$ hybrids with the degree of $\mathrm{A}$ or B-type depending on the direction of the oscillating dipole [12]. All out-of-plane vibrations will have C-type bands with strong $\mathrm{Q}$ branches and relatively weak $\mathrm{P}$ and $\mathrm{R}$ branches. The PR-branch separations of trans-vinyl ketene are calculated to be $13.5,11.3$, and $20.3 \mathrm{~cm}^{-1}$ for the pure A, B, and C-type bands, respectively, using the formulas of Seth-Paul [12]. For the cisrotamer the PR-branch separations are calculated to be $15.9,13.3$, and $23.9 \mathrm{~cm}^{-1}$, respectively, for A, B, and C-type bands. The observed band contours are all either A/B-hybrids with PR-branch separations in the range $11.5-12.5 \mathrm{~cm}^{-1}$ or C-type bands that are partially resolved. The spacing of the fine structure in these bands is ca. $2.4 \mathrm{~cm}^{-1}$, which is close to $2 \mathrm{~A}$ of trans-vinyl ketene, but about $5 \mathrm{~A}$ for the cis-rotamer. Thus, all the strongest bands clearly belong to the trans-rotamer. The wavenumbers of the central minima or of the dominant Q-branches of the absorption bands in Fig. 3 are listed in Table 5 .

Vinyl ketene has 21 normal vibrations of which 15 are in-plane vibrations (symmetry species a') and 6 are out-of-plane vibrations (species $\mathrm{a}^{\prime \prime}$ ). By analogy to vinyl allene [4] and ketene [10] four vibrations (the $\mathrm{C}=\mathrm{C}=\mathrm{O}$ in-plane bend, two skeletal in-plane bends, and the vinyl torsion) can be expected to have wavenumbers below the range covered. In fact, it is clearly seen in the microwave spectrum, Fig. 2, that two vibrations have wave-

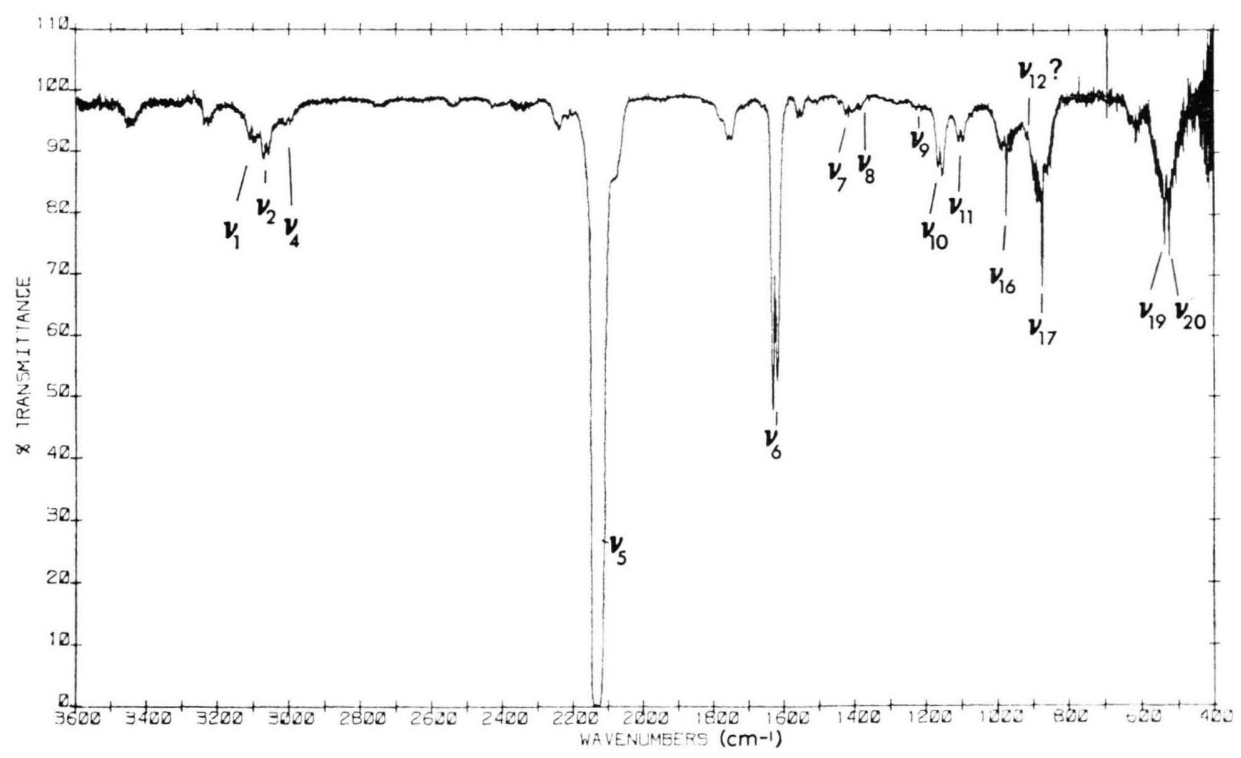

Fig. 3. The infrared gas spectrum of vinyl ketene at room temperature. Cell length $10 \mathrm{~cm}$, pressure ca. 4 Torr, resolution $1 \mathrm{~cm}^{-1}$. 
numbers so low that the excited states have significant populations at room temperature. A rough estimate from the relative intensities gives the lowest vibration, the vinyl torsion $v_{21}$, at ca. $105 \mathrm{~cm}^{-1}$ and the next lowest, an in-plane skeletal bend $\nu_{15}$, at ca. $155 \mathrm{~cm}^{-1}$. Since the $\mathrm{C}_{2}-\mathrm{H}_{1}$ and $\mathrm{C}_{3}-\mathrm{H}_{2}$ bonds are nearly antiparallel (Fig. 1), it may be expected that three of the six vibrations associated with them can be very weak in the infrared spectrum if they couple to form "in-phase" and "out-of-phase" vibrations. The assignment proposed in Table 5 is based on the band contours and on the assignments for vinyl allene [4], ketene [10] and 1,3-butadiene [13].

In the $\mathrm{C}-\mathrm{H}$ stretching region there are clearly three bands and possibly a fourth, but this is so overlapped that a band center cannot be determined. In the $\mathrm{C}-\mathrm{H}$ bending region all bands are weak or very weak and the assignments of $\nu_{8}$ and $v_{9}$ are rather questionable. The vinyl $\mathrm{CH}_{2}$ in-plane rocking vibration must be expected in the 850 to $1000 \mathrm{~cm}^{-1}$ region, but there are two relatively strong $\mathrm{C}$-type bands in this region and the presence of an A/B hybrid band is not clear. However, there is a slight extra dip between the two bands; this might be the central dip of an A/B-type band and is thus tentatively assigned to $v_{12}$. The two C-type bands are two of the three vinyl $\mathrm{C}-\mathrm{H}$ out-of-plane vibrations, but the third, which can be expected in the region $500-1100 \mathrm{~cm}^{-1}$, could not be located. This is to be expected due to the antiparallel arrangement of the $\mathrm{C}_{2}-\mathrm{H}_{1}$ and $\mathrm{C}_{3}-\mathrm{H}_{2}$ bonds as explained above. The two C-type bands at 526 and $540 \mathrm{~cm}^{-1}$ may be assigned to two ketene group out-of-plane vibrations.

In the spectrum are several weak or very weak bands that have been left unassigned. Some of these bands are in typical combination/overtone regions, while others may be fundamentals of cis-vinyl ketene or impurities.

\section{Conclusion and Discussion}

The microwave spectrum of vinyl ketene shows clearly the trans-rotamer, while no lines could be assigned to the cis-configuration. This indicates that the trans-vinyl ketene is much more abundant than the $c i s$-vinyl ketene. This is supported by the infrared spectrum, where all measurable PR-branch separations of $\mathrm{A} / \mathrm{B}$ hybrid bands are in the range
Table 5. Infrared transitions of vinyl ketene in the gas phase.

\begin{tabular}{|c|c|c|c|c|}
\hline $\begin{array}{l}\text { Wave- } \\
\text { number } \\
\left(\mathrm{cm}^{-1}\right)\end{array}$ & $\begin{array}{l}\text { Band } \\
\text { type }\end{array}$ & $\begin{array}{l}\text { Relative } \\
\text { intensity }{ }^{a}\end{array}$ & $\begin{array}{l}\text { Assign- } \\
\text { ment }^{b}\end{array}$ & $\begin{array}{l}\text { Approximate } \\
\text { description }\end{array}$ \\
\hline 3448 & $\mathrm{~A} / \mathrm{B}$ & $\mathbf{w}$ & & \\
\hline 3230 & $\mathrm{~A} / \mathrm{B}$ & w & & \\
\hline 3106 & $\mathrm{~A} / \mathrm{B}$ & $\mathbf{w}$ & $v_{1}$ & \\
\hline 3065 & $\mathrm{~A} / \mathrm{B}$ & $\mathrm{w}$ & $\nu_{2}$ & olefinic C-H str. \\
\hline 3003 & $\mathrm{~A} / \mathrm{B}$ & vw & $v_{4}$ & \\
\hline 2750 & $\mathrm{~A} / \mathrm{B}$ & vw & & \\
\hline 2535 & $\mathrm{~A} / \mathrm{B}$ & $\mathbf{v w}$ & & \\
\hline 2420 & $\mathrm{~A} / \mathrm{B}$ & vw & & \\
\hline 2245 & $\mathrm{~A} / \mathrm{B}$ & vw & & \\
\hline 2207 & $\mathrm{~A} / \mathrm{B}$ & vw & $2 v_{11}$ & $2 * 1102=2204$ \\
\hline 2137 & $\mathrm{~A} / \mathrm{B}$ & vs & $v_{5}$ & ketene $\mathrm{C}=\mathrm{O}$ str. \\
\hline 2090 & sh, A/B & w & & $\nu_{5}$ cis $?$ \\
\hline 1785 & $\mathrm{sh}$ & vw & & \\
\hline 1755 & $\mathrm{~A} / \mathrm{B}$ & $\mathbf{w}$ & $2 v_{17}$ & $2 * 875=1750$ \\
\hline 1720 & sh, A/B & $\mathrm{vw}$ & & \\
\hline 1626 & $\mathrm{~A} / \mathrm{B}$ & $\mathrm{s}$ & $v_{6}$ & vinyl $\mathrm{C}=\mathrm{C}$ str. \\
\hline 1557 & $\mathrm{~A} / \mathrm{B}$ & $\mathrm{w}$ & & \\
\hline 1418 & $\mathrm{~A} / \mathrm{B}$ & w & $v_{7}$ & vinyl $\mathrm{CH}_{2}$ scissors \\
\hline 1392 & $\mathrm{~A} / \mathrm{B}$ & $\mathrm{vw}$ & $v_{8}$ & $=\mathrm{C}-\mathrm{C}=$ str. \\
\hline 1220 & $\mathrm{~A} / \mathrm{B}$ & $\mathrm{vw}$ & $\nu_{9} ?$ & $\mathrm{C}-\mathrm{H}$ bend \\
\hline 1161 & $\mathrm{~A} / \mathrm{B}$ & $\mathrm{m}$ & $v_{10}$ & $\begin{array}{l}\mathrm{C}-\mathrm{H} \text { (ketene) } \\
\text { bend? }\end{array}$ \\
\hline 1102 & $\mathrm{~A} / \mathrm{B}$ & w & $v_{11}$ & ketene $\mathrm{C}=\mathrm{C}$ str. \\
\hline 976 & C & $\mathrm{m}$ & $v_{16}$ & vinyl twist \\
\hline $915 ?^{\mathrm{c}}$ & $\mathrm{A} / \mathrm{B}$ & & $v_{12} ?$ & vinyl $\mathrm{CH}_{2}$ rock \\
\hline 875 & $\mathrm{C}$ & $\mathrm{m}$ & $v_{17}$ & vinyl $\mathrm{CH}_{2}$ wag \\
\hline $\begin{array}{l}633 ? ?^{\mathrm{d}} \\
615 ?^{\mathrm{d}}\end{array}$ & & $\begin{array}{l}\text { vw } \\
\text { vw }\end{array}$ & & $v_{9}(\mathrm{e})$ propyne \\
\hline $\begin{array}{l}615 ? ?^{\alpha} \\
540\end{array}$ & $\begin{array}{l}\mathrm{A} / \mathrm{D} \\
\mathrm{C}\end{array}$ & $\mathrm{m}$ & $\nu_{19}$ & ketene $\mathrm{C}-\mathrm{H}$ out- \\
\hline 526 & $\mathrm{C}$ & $\mathrm{m}$ & $v_{20}$ & $\begin{array}{l}\text { of-plane bend } \\
\mathrm{C}=\mathrm{C}=\mathrm{O} \text { out-of- } \\
\text { plane bend }\end{array}$ \\
\hline ca. $155^{\mathrm{e}}$ & - & - & $v_{15}$ & $\begin{array}{l}\text { in plane } \\
\text { skeletal bend }\end{array}$ \\
\hline ca. $105^{\mathrm{e}}$ & - & - & $v_{21}$ & vinyl torsion \\
\hline
\end{tabular}

a $\mathrm{vs}=$ very strong; $\mathrm{s}=$ strong, $\mathrm{m}=$ medium, $\mathrm{w}=$ weak, $\mathrm{vw}=$ very weak.

b Question marks at uncertain assignments.

c The existence of this band is very uncertain.

d Possibly the strongest band in propyne, $v_{9}(\mathrm{e})$, overlapping an $\mathrm{A} / \mathrm{B}$ hybrid band from vinyl ketene.

e Estimated from the microwave spectrum. The uncertainty is $30 \mathrm{~cm}^{-1}$.

expected for the trans-rotamer and about $1 \mathrm{~cm}^{-1}$ less than the smallest separation expected for the cis-rotamer. Further, the spacings in the partially resolved C-type bands are of the magnitude expected for trans-vinyl ketene but much too large for cis-vinyl ketene. Thus, all the strongest infrared bands can be assigned to the trans conformation, while there are no positive assignments to the cis form. If it is assumed that the low frequency shoulder on $v_{5}$, the very strong ketene band at $2137 \mathrm{~cm}^{-1}$, is the $v_{5}$ of the cis-rotamer, then from 
the infrared spectrum Fig. 3 a rough estimate for the trans: cis abundance is $10: 1$ at room temperature. This is an upper limit for the cis-conformation.

The dipole moment of trans-vinyl ketene was found to be $0.987(23) D$. This is surprisingly smaller than in ketene $(1.414(10) D)[14]$ and methyl ketene $(1.790(15) D)$ [9].

Of the 21 normal vibrations of vinyl ketene four are not observed, two have been roughly estimated from the microwave spectrum and three are uncertain. A complete vibrational assignment will first be possible with the knowledge of the infrared spectrum of vinyl ketene in the condensed phase, of the far-infrared spectrum, and preferably of the Raman spectrum.

Note added in proof: It has been brought to my attention that R. D. Godfrey and M. Woodruff at Monash University, Australia, in an independent study have observed the microwave spectra of vinyl ketene in the pyrolysis products of vinylacetic anhydride. A report onthis will appear shortly.

[1] R. W. Holder, H. S. Freiman, and M. S. Stefanchik, J. Org. Chem. 41, 3303 (1976).

[2] G. Rousseau, R. Bloch, P. Le Perchec, and J.M. Conia, J. C. S., Chem. Comm. 1973, 795.

[3] J. K. Terlouw, P. C. Burgers, and J. L. Holmes, J. Amer. Chem. Soc. 101, 225 (1979).

[4] P. Klaboe, T. Torgrimsen, D. H. Christensen, H. Hopf, A. Eriksson, G. Hagen, and S. J. Cyvin, Spectrochim. Acta 30 A, 1527 (1974).

\section{Acknowledgements}

Support by the Alexander von Humboldt Foundation in the form of a fellowship is gratefully acknowledged. Thanks are due to Dr. G. Winnewisser, Max-Planck-Institute for Radioastronomy, for letting me use the microwave spectrometer, to Dr. Koichi Yamada for access to his centrifugal distortion fitting program, to Drs. Manfred and Brenda Winnewisser for continuous interest in this work and comments on the manuscript, and to Dr. Jürgen Vogt who recorded some of the infrared spectra*.

[5] A. Bouchy and G. Roussy, J. Mol. Spectrosc. 68, 156 (1977).

[6] R. G. Ford, J. Mol. Spectrosc. 65, 273 (1977).

[7] J. S. Muenter, J. Chem. Phys. 48, 4544 (1968).

[8] J. K. G. Watson, J. Chem. Phys. 46, 1935 (1967).

[9] B. Bak, J. J. Christiansen, K. Kunstmann, L. Nygaard, and J. Rastrup Andersen, J. Chem. Phys. 45, $883(1966)$

[10] C. B. Moore and G. C. Pimentel, J. Chem. Phys. 38, 2816 (1963).

[11] W. J. Potts and R. A. Nyquist, Spectrochim. Acta 15, 679 (1959).

[12] W. A. Seth-Paul, J. Mol. Struct. 3, 403 (1969).

[13] Yu. N. Panchenko, Spectrochim. Acta 31 A, 1201 (1975).

[14] H. R. Johnson and M. W. P. Strandberg, J. Chem. Phys. 20, 687 (1952). 